\title{
ADAM10 overexpression in human non-small cell lung cancer correlates with cell migration and invasion through the activation of the Notch1 signaling pathway
}

\author{
JIANLI GUO ${ }^{1}$, LEI HE ${ }^{2}$, PING YUAN ${ }^{1}$, PENG WANG ${ }^{1}$, YANJUN LU ${ }^{3}$, FANGLI TONG ${ }^{1}$, \\ YU WANG ${ }^{1}$, YANHUA YIN ${ }^{1}$, JUN TIAN ${ }^{1}$ and JUN SUN ${ }^{1}$
}

${ }^{1}$ Department of Biochemistry and Molecular Biology, Tongji Medical College, ${ }^{2}$ Department of Blood Transfusion and

${ }^{3}$ Clinical Laboratory, Tongji Hospital, Tongji Medical College, Huazhong University

of Science and Technology, Wuhan 430030, P.R. China

Received June 13, 2012; Accepted August 6, 2012

DOI: 10.3892/or.2012.2003

\begin{abstract}
A disintegrin and metalloproteinase 10 (ADAM10) was identified as a key protease in the ectodomain shedding of various substrates, such as Notch1 protein,ErbB2 and E-cadherin, which are important in the development of non-small cell lung cancer (NSCLC). The aim of this study was to investigate the role of ADAM10 in NSCLC metastasis.We characterized the expression of ADAM10 and Notch1 in human NSCLC tissues in vivo. Immunohistochemical analysis indicated that ADAM10 expression was significantly increased in the NSCLC tissues, particularly in the metastatic tissues. Futhermore, ADAM10 overexpression positively correlated with Notch1 expression in the NSCLC tissues. The in vitro downregulation of ADAM10 expression using ADAM10 short hairpin RNA (shRNA) reduced the migration and invasion of NSCLC cells. We present further evidence that ADAM10 promotes NSCLC cell migration and invasion via the activation of the Notch1 signaling pathway. Taken together, our results suggest that ADAM10 may serve as a potential target for the therapeutic intervention of NSCLC metastasis. The data provided in this study may aid in the further understanding of the function of ADAM10 in the progression of NSCLC and open new perspectives for the diagnosis and treatment of NSCLC.
\end{abstract}

\section{Introduction}

Lung cancer is the leading cause of cancer-related mortality not only in China, but worldwide. Non-small cell lung cancer (NSCLC) accounts for $80 \%$ of lung cancer cases with a 5-year survival rate of $16 \%$ (1). Surgical resection of primary NSCLC

Correspondence to: Dr Jun Sun, Department of Biochemistry and Molecular Biology, Tongji Medical College, Huazhong University of Science and Technology, 13 Hangkong Road, Wuhan 430030, P.R. China

E-mail: sunjun99@hotmail.com

Key words: a disintegrin and metalloproteinase 10, non-small cell lung cancer, metastasis, Notch1 is frequently followed by tumor recurrence at distant sites as a result of occult micrometastasis (2). Despite improvements in diagnosis and treatment, the overall survival for lung cancer patients is disappointing and the majority of deaths are associated with metastasis to the brain, lung and bones (3). Thus, there is an urgent need to further understand the molecular mechanisms of NSCLC progression and to discover new molecular targets for treatment.

Proteolytic ectodomain release, a process known as 'shedding', has been recognized as a key mechanism for regulating the function of a diversity of cell surface proteins. A disintegrin and metalloproteases (ADAMs) have emerged as the major proteinase family that mediates ectodomain shedding. ADAMmediated shedding is essential for a number of biological processes, such as cell fate determination, cell migration, wound healing, cell proliferation and angiogenesis (4). Moreover, numerous members of the ADAM family such as ADAM9, 10 and 17 have been proven to be associated with cancer cell proliferation, migration and invasion (5), implying that ADAMs play pivotal roles in cancer formation and progression. A recent study has also highlighted the potential of targeting ADAM family members as a new approach for anticancer therapy (6). For cancer metastasis, it has been demonstrated that the overexpression of ADAM9 in NSCLC correlates with brain metastasis (7). ADAM17 plays an important role in hepatocellular carcinoma metastasis (8). ADAM10 silencing or rhADAM10 have been shown to have no effect on cell viability but markedly reduce the invasiveness and migration of pancreatic cancer cells (9). ADAM10 has been demonstrated as one of the key molecules in several of the shedding events characterized to date and has also been implicated in the shedding of a number of substrates that drive cancer progression, including the Notch receptor, EGF, ErbB2, E-cadherin, L1-CAM and inflammatory cytokines (10). Previous studies have shown that ADAM10 is overexpressed in a variety of human cancers, such as pancreatic, oral squamous cell carcinoma, ovarian and uterine cancers $(9,11,12)$. In addition, certain in vitro studies have revealed that the downregulation of ADAM10 with specific small interfering RNA (siRNA) results in the reduced migration and invasion of cancer cells via the inhibition of the 
cleavage of substrates, such as CLCX-16 and L1-CAM $(9,13)$. With respect to lung cancer, a previous report indicated that the expression of the active form of ADAM10 was increased in NSCLC tissues and it has been hypothesized that ADAM10 may be involved in NSCLC tumor angiogenesis and/or metastasis (14). Despite the abovementioned reports, the involvement of ADAM10 in human lung cancer remains unclear.

It has previously been described that several prominent substrates of ADAM10 involving different signaling pathways, such as Notch receptor, ErbB2/ERK and E-cadherin/ $\beta$-catenin play important roles in the initiation and/or progression of lung cancer (15-17). Notch proteins, the transmembrane receptors, are highly conserved in the development and the determination of cell fate. To date, 4 mammalian transmembrane receptors (Notch1-4) and 5 transmembrane ligands (Jagged1, 2, $\delta 1,3$ and 4) have been identified (18). Recently it has been increasingly recognized that the Notch signaling pathway plays an important role in the initiation and/or progression of lung cancer $(15,19,20)$. Furthermore, growing evidence suggests that Notch1 promotes the proliferation, invasion and migration of lung cancer cells through the activation of the signaling pathway $(21,22)$. However, the underlined mechanisms need to be further investigated. Clinical data have demonstrated that $30 \%$ of NSCLC cases have increased Notch1 activity and $10 \%$ of NSCLC cases have gain-of-function mutations on the Notch1 gene (23). In NSCLC adenocarcinoma, tumor cell Notch1 and VEGF expression have been shown to be independently associated with poor prognosis (24). In mammals, both ADAM10 and ADAM17 cleave Notch under certain situations; however, the embryos of ADAM10 knockout mice have shown a reduced or disorganized expression of Notch target genes and enhanced levels of Notch protein, indicating a lack of cleavage/ activation (25). To date, there is no evidence regarding the lack of Notch signaling or processing in ADAM17 knockout mice. As a result, we hypothesized that ADAM10 promotes NSCLC progression via the cleavage of its substrate, the Notch1 receptor, and that the specific targeting of ADAM10 represents a more effective strategy for NSCLC cancer therapy compared with the traditional treatment. The aim of this study was to investigate the involvement of ADAM10 in NSCLC progression and to further explore the potential mechanisms of the biological behavioral changes affected by ADAM10 in NSCLC cells.

In the present study, we examined the protein expression of ADAM10 and Notch1 in NSCLC tissues and corresponding normal tissues. The results showed that the expression of ADAM10 was upregulated in NSCLC tissues and that the elevated expression of ADAM10 significantly correlated with NSCLC metastasis. In addition, there was positive correlation between the expression of ADAM10 and Notch1 in NSCLC tissues. We provide evidence that the knockdown endogenous expression of ADAM10 by RNA interference may inhibit A549 NSCLC cell migration and invasion, and that this anticancer effect of silencing ADAM10 expression may target the Notch1 signaling pathway.

\section{Materials and methods}

Reagents and antibodies. G418 and 3-(4,5-dimethylthiazol2-yl)-2,5-diphenyltetrazolium bromide (MTT) were purchased from Sigma (St. Louis, MO, USA). The $\gamma$-secretase inhibitor
(DAPT) was purchased from Calbiochem (San Diego, CA, USA) and used at a concentration of $10 \mu \mathrm{mol} / 1$. The antibody against human ADAM10 was purchased from eBioscience (San Diego, CA, USA). Antibodies to the $\mathrm{C}$ terminal of human Notch1/NICD and $\beta$-actin were obtained from Santa Cruz Biotechnology (Santa Cruz, CA, USA). Anti-human ERK1/2 and p-ERK1/2 were from Cell Signaling Technology, Inc. (Beverly, MA, USA). Anti-human $\beta$-catenin antibody was purchased from R\&D Systems (Minneapolis, MN, USA).

Clinical tumor specimens. Clinical NSCLC specimens were collected between December 2009 and October 2010 at the Tongji Hospital (Wuhan, China). For each patient, a sample of adjacent and apparently non-affected tissue was also obtained and used as the normal control. All specimens from patients who underwent surgery were formalin-fixed and paraffin-embedded for histopathological diagnosis and immunohistochemical analysis. The histological diagnosis of each tumor was confirmed on hematoxylin and eosin-stained sections. Histological classification was assessed in all patients according to the World Health Organization International Histological Classification of Tumors (18). The clinical features of the patients are presented in Table I. All patients provided informed consent for the excess pathological specimens obtained for research purposes. Research was carried out in compliance with the Helsinki Declaration and the approval of the Ethics Committee of Tongji Medical College.

Immunohistochemical (IHC) analysis and semi-quantitative evaluation. The antibody staining against ADAM10 or Notch1 was performed on 4- $\mu \mathrm{m}$ histological sections of formalin-fixed, paraffin-embedded tumor and adjacent normal samples as previously described (19). For each spot, areas of most intense and/or predominant staining pattern were scored by eye. The immunostaining pattern for each case was independently evaluated by 3 investigators (P.W., Y.L. and Y.W.) using a two-headed microscope. The cells with brown staining were considered positively stained. Immunostaining for markers was classified by a semiquantitative method based on a scale that takes into account the intensity and distribution of the staining: staining intensity was categorized as 0 (negative), 1 (weak), 2 (moderate) or 3 (strong). Staining areas were scored as 0 (no positive cells), 1 ( $\leq 25 \%$ positive cells), 2 ( $>25 \%$ and $\leq 50 \%$ positive cells) and 3 ( $>50 \%$ positive cells). To gauge both the staining intensity and distribution simultaneously, the average values of intensity for each tissue were multiplied by the average values for percentage area stained in each tissue to derive a composite histoscore (histoscore $=$ percentage area stained $\mathrm{x}$ staining intensity). For example, a tissue with intense, uniform staining was assigned the maximum histoscore of 9. For statistical analysis, the histoscore of each section no $<6$ was classified as high expression (histoscore 6-9) and low expression cases included both negative (histoscore 0 ) and weakly positive cases (histoscore 1-4). Assigning a histoscore is now a commonly used method for evaluating both staining uniformity and intensity in tissues in order to better correlate results between multiple samples from immunohistochemical analysis (19).

Cell culture and stable ADAM10 knockdown. The human NSCLC cell line, A549, purchased from the China Center for 
Table I. Correlation between ADAM10 expression and clinicopathological factors in tumor tissues from 56 patients with NSCLC.

\begin{tabular}{|c|c|c|c|c|}
\hline \multirow[b]{2}{*}{ Factors } & \multirow[b]{2}{*}{$\mathrm{n}$} & \multicolumn{2}{|c|}{ ADAM10 expression } & \multirow[b]{2}{*}{ P-value } \\
\hline & & Low histoscore $(0-4)$ & High histoscore (6-9) & \\
\hline \multicolumn{5}{|l|}{ Age (years) } \\
\hline$<60$ & 34 & 16 & 18 & \multirow[t]{2}{*}{ NS (0.65) } \\
\hline$\geq 60$ & 22 & 9 & 13 & \\
\hline \multicolumn{5}{|l|}{ Gender } \\
\hline Male & 41 & 18 & 23 & \multirow[t]{2}{*}{ NS $(0.85)$} \\
\hline Female & 15 & 7 & 8 & \\
\hline \multicolumn{5}{|l|}{ Tumor size $(\mathrm{cm})$} \\
\hline$<5$ & 30 & 16 & 14 & \multirow[t]{2}{*}{ NS (0.16) } \\
\hline$\geq 5$ & 26 & 9 & 17 & \\
\hline \multicolumn{5}{|l|}{ Histological type } \\
\hline Squamous cell carcinoma & 27 & 13 & 14 & \multirow[t]{3}{*}{ NS $(0.68)$} \\
\hline Adenocarcinoma & 25 & 11 & 14 & \\
\hline Adenosquamous cell carcinoma & 4 & 1 & 3 & \\
\hline \multicolumn{5}{|l|}{ Histological differentiation } \\
\hline Poor & 13 & 3 & 10 & \multirow[t]{3}{*}{$0.045^{\mathrm{a}}$} \\
\hline Moderate & 25 & 10 & 15 & \\
\hline Well & 18 & 12 & 6 & \\
\hline \multicolumn{5}{|l|}{ Lymph node metastasis } \\
\hline Negative & 18 & 13 & 5 & \multirow[t]{2}{*}{$0.004^{\mathrm{a}}$} \\
\hline Positive & 38 & 12 & 26 & \\
\hline \multicolumn{5}{|l|}{ Distant metastasis } \\
\hline Negative & 37 & 21 & 16 & \multirow[t]{2}{*}{$0.011^{\mathrm{a}}$} \\
\hline Positive & 19 & 4 & 15 & \\
\hline
\end{tabular}

Statistical analysis was performed using the $\chi^{2}$ test. ${ }^{\mathrm{P}} \mathrm{P}<0.05$ was considered significant. NS, not significant; ADAM10, a disintegrin and metalloproteinase, NSCLC, non-small cell lung cancer.

Type Culture Collection (CCTCC; Wuhan, China), was cultured according to the guidelines provided and passaged in our laboratory for $<6$ months after receipt.

Three short hairpin (sh)RNA oligonucleotides were synthesized to target 3 different regions in human ADAM10 cDNA: 1, GGGTCTGTTATTGATGGAAGA; 2, GCTGATGAGAA GGACCCTACA; and 3, GCATACACAAGTGTGCATTAA. They were cloned respectively into shRNA expression vector, pGenesil-4, with U6 promoter (Genesil Corp., Wuhan, China), containing selectable marker GFP to facilitate the selection of stably transfected cells. The recombinant shADAM10 and control shCon plasmids were transfected into A549 cells using Lipofectamine $^{\mathrm{TM}} 2000$ (Invitrogen, Carlsbad, CA, USA). The cells were then screened with G418 $(800 \mu \mathrm{g} / \mathrm{ml})$, and the stably transfected cell clones were picked after 3 weeks and the silencing effect was detected by western blot analysis.

Scratch wound assay. The selected A549 cells were plated in 6 -well plates at a concentration of $3 \times 10^{5}$ cells/well. Approximately $48 \mathrm{~h}$ later, the confluent cell layers were carefully scratched using sterile pipette tips. Non-adherent cells and cellular debris were removed by washing with phosphatebuffered saline (PBS). Cells were observed under a microscope and digitally photographed at different times. Inhibition of cell migration was assessed when the wound in the control was closed and quantified by using a measuring tool in Photoshop CS2 (Adobe Systems, Inc., USA).

Cell invasion assay. Cell invasion was examined using Transwell chambers $(8.0 \mu \mathrm{m})$ according to the manufacturer's instructions (Costar, USA) and the top chambers were coated with Matrigel (BD Biosiences, USA). The cells were transferred to the top chamber of each prepared Transwell chamber at a density of $4 \times 10^{5}$ cells $/ \mathrm{ml}(100 \mu \mathrm{l})$. The bottom chamber contained DMEM supplemented with $10 \%$ FBS. The cells were allowed to migrate for $24 \mathrm{~h}$ at $37^{\circ} \mathrm{C}$. Non-invading cells were removed from the top surfaces with a cotton swab. The membranes were fixed in $95 \%$ ethanol and stained with $0.1 \%$ crystal violet. The cells that had penetrated to the bottom surface of each membrane were counted with 10 random fields on each microscope slide.

Western blot analysis. The cell pellet was lysed in buffer containing $10 \mathrm{mmol} / \mathrm{l}$ Tris- $\mathrm{HCl}(\mathrm{pH} 8.0), 1 \mathrm{mmol} / 1 \mathrm{MgCl}_{2}$, $1 \% \mathrm{NP}-40,0.5 \%$ sodium deoxycholate, $1 \mathrm{mmol} / \mathrm{l}$ phenylmethylsulfonyl fluoride, $1 \mathrm{mg} / \mathrm{l}$ aprotinin and $0.02 \mathrm{mg} / \mathrm{l}$ 
leupeptin. Following cell protein quantification, $60 \mu \mathrm{g}$ of protein were subjected to $7.5 \%$ SDS-PAGE. The proteins separated on the PAGE gel were transferred onto nitrocellulose membranes, which were blocked for $2 \mathrm{~h}$ at room temperature. The membranes were then incubated overnight at $4^{\circ} \mathrm{C}$ with primary antibodies, depending on the assays, which were anti-human antibodies against ADAM10, Notch1/NICD, ERK1/2,p-ERK1/2, $\beta$-catenin and $\beta$-actin. After being washed 3 times with TBST $(50 \mathrm{mmol} / \mathrm{l}$ Tris- $\mathrm{HCl} \mathrm{pH}$ 7.6, $150 \mathrm{mmol} / \mathrm{l}$ $\mathrm{NaCl}, 0.1 \%$ Tween-20), the membranes were incubated with secondary antibodies conjugated with horseradish peroxidase, and then washed 3 times with TBST again. Immunoreactive bands were visualized by an enhanced chemiluminescence (ECL) system and the level of protein expression was determined using Image Quant TL software (Amersham Pharmacia Biotech, USA).

Immunofluorescence. Cells were plated on glass coverslips in 6-well culture plates. After $24 \mathrm{~h}$, the cells were fixed with $4 \%$ paraformaldehyde for $15 \mathrm{~min}$. Subsequently, the cells were permeabilized with $0.1 \%$ Triton X-100 for $15 \mathrm{~min}$ at room temperature, washed with PBS and blocked with PBS containing $0.5 \%(\mathrm{w} / \mathrm{v})$ bovine serum albumin (BSA) and $0.15 \%$ $(\mathrm{w} / \mathrm{v})$ glycine (BSA buffer) for $1 \mathrm{~h}$ at room temperature. Cells were treated with anti-Notch1/NICD antibody (1:50 dilution in BSA buffer) for $1 \mathrm{~h}$ at room temperature. Cells were then washed with BSA buffer and incubated with secondary antibody conjugated with Rhodamine for $1 \mathrm{~h}$ at room temperature. DAPI (200 ng/ml) was used for staining the nuclei. Images were acquired by a Nikon fluorescence microscope, with NIS-Elements 3.1 software.

Statistical analysis. The direction and strength of the association between ADAM10 and Notch1 were evaluated and compared with the Spearman correlation test. Experimental differences were examined for statistical significance using the $\chi^{2}$ or Student's t-test. Data are reported as the means \pm SD and a P-value $<0.05$ was considered to indicate a statistically significant difference. All statistical analyses were performed with the Superior Performance Software System (SPSS) 15.0 for Windows (SPSS, Inc., Chicago, IL, USA).

\section{Results}

Expression of ADAM10 protein in NSCLC clinical specimens. In order to investigate the potential role of ADAM10 expression in NSCLC progression, immunohistochemical analysis was carried out to evaluate the endogenous protein expression in the paraffin-embedded tumor and adjacent normal tissues. Fig. 1A illustrates the ADAM10 protein expression in 2 representative patients. Through statistical analysis of ADAM10 protein expression in NSCLC tissues from 56 patients, we found that the histoscore of ADAM10 was statistically increased in tumor tissues compared to normal tissues $(\mathrm{P}<0.01$, Fig. 1B). Furthermore, the strong immunoreactivity of ADAM10 expression was displayed in both lymph node and distant metastatic NSCLC samples (Fig. 1C). Compared with non-metastatic tumor tissues (Fig. 2A), the statistical data revealed that the ADAM10 expression levels were significantly elevated in the metastatic tissues $(\mathrm{P}<0.05$; Fig. 1D). These find- ings suggest a possible link between ADAM10 overexpression and metastasis in NSCLC.

To determine the clinical significance of ADAM10 expression in NSCLC, the correlation of its expression pattern in patient tumor samples with clinical features was analyzed. The results are summarized in Table I. Among patients with NSCLC, a high ADAM10 expression was not associated with age, gender, tumor size and histological types. However, the overexpression of ADAM10 showed a significant correlation with poor differentiation, lymph node and distant metastasis $(\mathrm{P}<0.05)$. These results further suggest that the overexpression of ADAM10 correlates with the aggravated progression of NSCLC, particularly with metastasis. This protein may be a clinically significant player in NSCLC metastasis.

Knockdown of ADAM10 inhibits the migration and invasion of A549 NSCLC cells. To directly examine whether ADAM10 is functionally important for NSCLC metastasis, ADAM10 shRNA was used to silence ADAM10 expression in A549 cells, a highly metastatic NSCLC cell line with a high expression of ADAM10 (Fig. 2A). As shown in Fig. 2A, 3 A549-derived stable cell lines were selected that expressed different shRNA, and only 1 shRNA construct targeting ADAM10 (shADAM10-1) effectively inhibited its expression at the protein level, thus prompting us to select this A549 stable cell line for further study.

The migration and invasion of tumor cells are usually considered a prerequisite of tumor metastasis. To assess whether the downregulation of ADAM10 in A549 cells reduces cell migration, a wound healing migration assay was performed. After $24 \mathrm{~h}$, a significant delay in the wound closure rate was observed with the microscopic examination of shADAM10-1 cells compared to the control cells $(\mathrm{P}<0.01$; Fig. 2B).

To examine the influence of silencing ADAM10 expression on the inhibition of NSCLC cell invasion, standardized Matrigel invasion assay was employed. As was expected, the invasion number of shADAM10-1 cells was significantly less compared to the control cells $(\mathrm{P}<0.05$; Fig. $2 \mathrm{C})$. These results suggest that the repression of ADAM10 expression in NSCLC cells inhibits cellular migration and invasion. As a consequence, ADAM10 plays a pivotal role in NSCLC metastasis.

Silencing ADAM10 expression inhibits the cleavage of the intracellular domain of Notchl in A549 NSCLC cells. To explain the above effect of ADAM10 on the migration and invasion of NSCLC, we selectively analyzed several signaling factors which are related to NSCLC metastasis. Previous studies have shown that the ERBB/ERK, E-cadherin/ $\beta$-catenin and Notch1 signaling pathways play key roles in NSCLC formation and metastasis progression. Of note, the proteolytic activated function of ADAM10 has been implicated in these pathways (10). In line with this, the ADAM10 regulation of ERK $1 / 2$ and $\beta$-catenin expression was first investigated by western blot analysis. As a result, the stable silencing of ADAM10 failed to suppress $\beta$-catenin expression and ERK1/2 phosphorylated activation in A549 cells (Fig. 3A). Thus, additional consideration was given to the possible role of ADAM10 in Notch1 signaling due to its proteolytic function contributing to the cleavage of Notch1 to release the Notch1 intracellular domain (NICD), the activated form of Notch1 
A Normal tissue

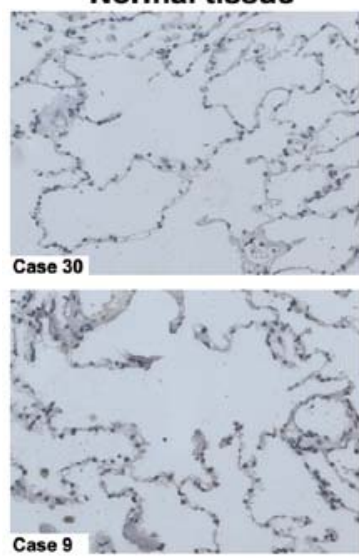

C

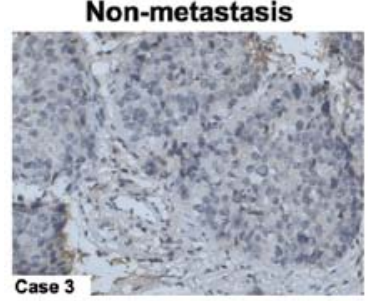

Tumor tissue

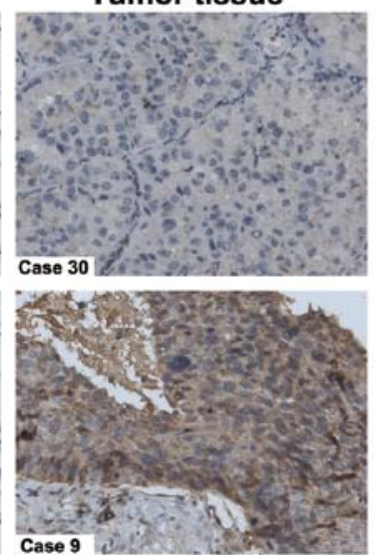

Lymph node metastasis

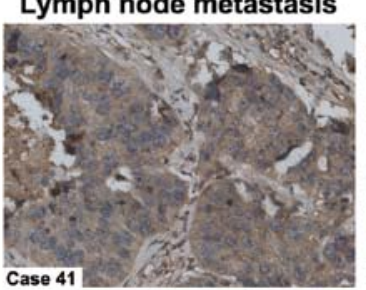

B

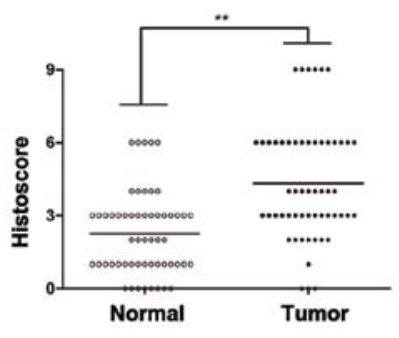

ADAM10 expression in tissue

Figure 1. Expression of the ADAM10 protein in NSCLC clinical specimens. (A) Immunohistochemical analysis of ADAM10 in paired paraffin-embedded human NSCLC tissues and the adjacent normal ones. ADAM10 staining in 2 representative cases, the cells with brown staining were considered positively stained, ADAM10 expression showed predominately membranous and cytoplasmic localization. Normal tissues (left panels) showed negative or weak staining, while tumor tissues showed moderate to strong ADAM10 staining (right panels). (B) Statistical analysis with histoscore (area stained multiplied by intensity) of ADAM10 staining in tissues from NSCLC patients $(\mathrm{n}=56)$. The ADAM10 expression levels were significantly higher in tumor tissues compared with non-affected normal ones. Original magnification, $\mathrm{x} 200 .{ }^{* *} \mathrm{P}<0.01$. (C) Immunohistochemical staining of ADAM10 in non-metastatic and metastatic malignant tissues from human NSCLC patients. Representative non-metastatic NSCLC tissues (left panel) showing weak membrane and cytoplasm ADAM10 staining (histoscore, 3). Carcinomas with lymph node and distant metastasis (middle and right) showing strong membrane and cytoplasm ADAM10 staining (histoscore, 6 and 9, respectively); (D) Statistical analysis revealed that the histoscores of ADAM10 were significantly elevated in metastatic carcinomas (including lymph node and distant metastasis, $n=38$ ) compared with non-metastatic tissues $(n=18)$. NM, non-metastasis; LM, lymph node metastasis; DM, distant metastasis. Original magnification, $\mathrm{x} 200 .{ }^{*} \mathrm{P}<0.05$.

A

B
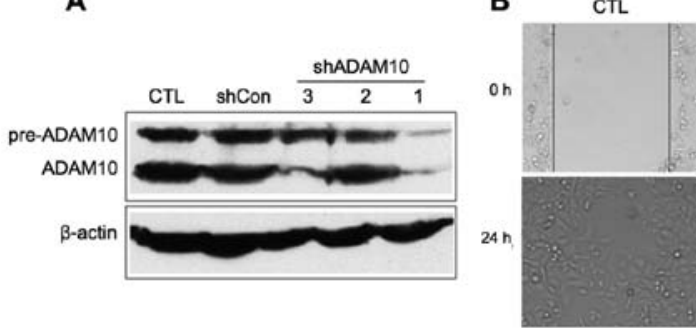

C
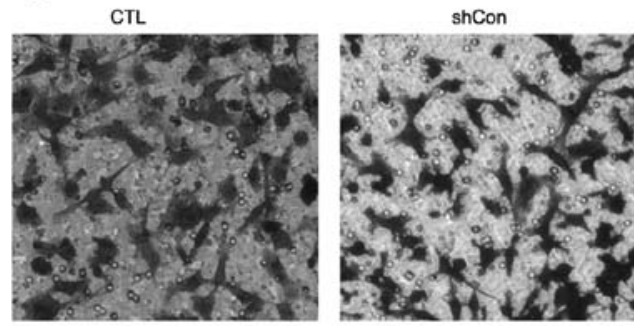

shCon

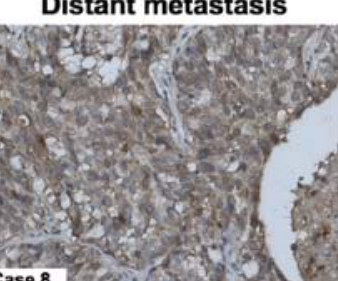

D

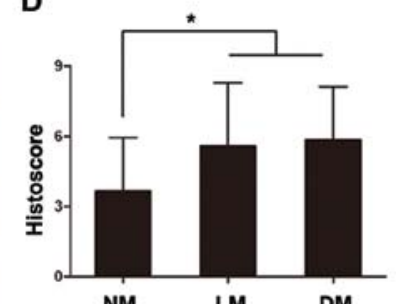


A

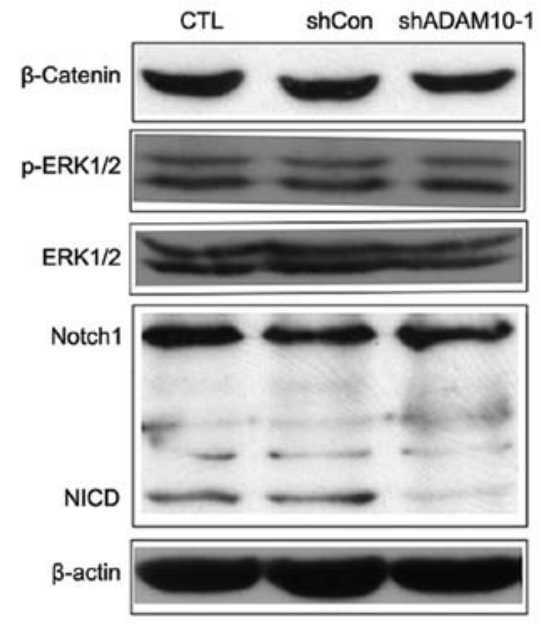

B

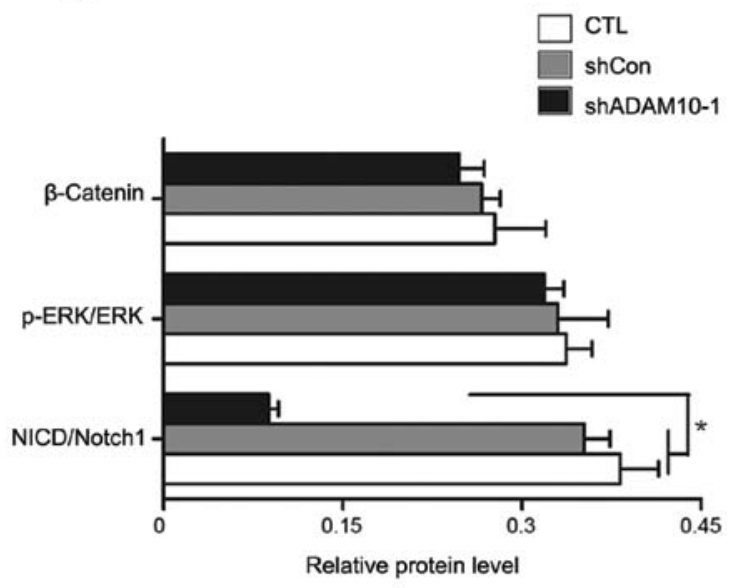

shCon

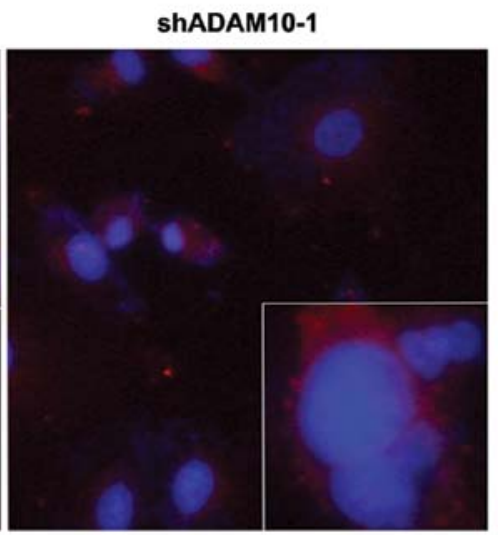

Figure 3. Abolished ADAM10 expression inhibits the release of the intracellular domain of Notch1 (NICD) in A549 NSCLC cells. (A) The effects of abolishment of ADAM10 on $\beta$-catenin protein expression, ERK and Notch1 signaling activation. Western blot analysis was used to analyze $\beta$-catenin, ERK1/2, p-ERK1/2 and Notch1 (including NICD) protein expression in the untreated A549 NSCLC (CTL), shADAM10-1 and shCon cells. $\beta$-actin served as the internal control. (B) Statistical analysis with relative protein levels of the abovementioned proteins in the indicated cell lines. Error bars indicate SD ( $\mathrm{n}=3$ ). " $\mathrm{P}<0.05$. (C) Fluorescence microscopy analysis for NICD in CTL, control and ADAM10 knockdown stable cell lines. Arrowheads indicate the expression of NICD in the nucleus. Red, NICD; blue, nuclear DNA. Original magnification, x200; x400 (insets).

that translocates to the nucleus to modulate the downstream gene expression to facilitate cancer progression (26). Of note, when the knockdown of ADAM10 occurred in A549 cells, the expression levels of NICD significantly decreased compared with the control cells (Fig. 3A and B). Consistently, the cellular immunofluorescence assay indicated the similar effect. As shown in Fig. 3C, the fluorescence intensity of NICD located in the nucleus was weakened in the shADAM10-1 cells. These results suggest that silencing ADAM10 expression in NSCLC cells inhibits the expression of NICD, the activated form of Notch1, and this effect may further inhibit downstream gene (such as Heyl) transcription (data not shown).

Notch1 signaling inhibitor suppresses the migration and invasion of A549 NSCLC cells. The above results suggest that the knockdown of ADAM10 suppresses metastasis through the inhibition of Notch1 activation in NSCLC cells. We then wished to examine whether the inhibition of Notch signaling can reduce NSCLC cell migration and invasion. To determine this, we examined the inhibition of Notch signaling with DAPT, a $\gamma$-secretase inhibitor (GSI) in A549 cells. Compared to the control cells, wound healing migration and Matrigel invasion assay revealed that the repression of Notch signaling by DAPT significantly decreased the migration and invasion rate of shADAM10-1 cells (Fig. 4). However, we did not observe any difference between the 2 groups treated with DAPT or shADAM10-1, respectively (Fig. 4). These results suggest that either ADAM10 siRNA or GSI inhibit the Notch1 signaling pathway, and further suppress the migration and invasion of NSCLC cells.

Significantly positive correlation between ADAMIO and Notchl expression in NSCLC. The above results indicated that the knockdown of ADAM10 inactivated the Notch1 signaling pathway via inhibition of NICD release from Notch1 in NSCLC cells, and then further suppressed downstream gene expression. This prompted us to investigate whether ADAM10 and Notch1 are co-expressed in NSCLC tissues. To determine this, the co-expression of ADAM10 and Notch1 was also detected with IHC in NSCLC samples. The strong immunostaining of ADAM10 correspondingly displayed a high expression of Notch1 in the same NSCLC sample, which was overexpressed 
A
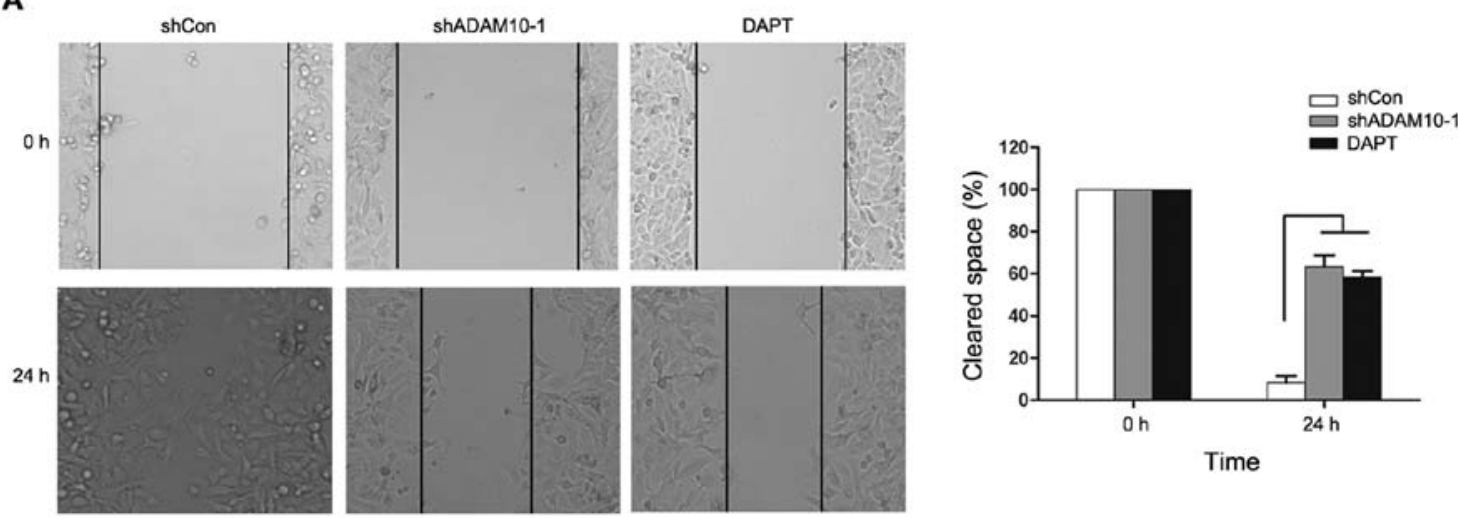

B
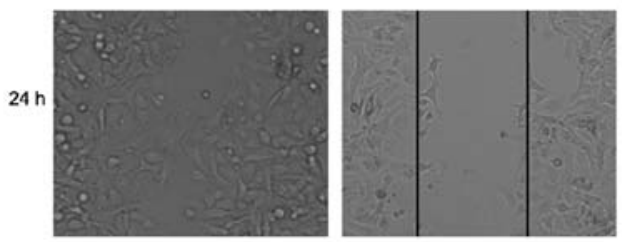

shCon

ShADAM10-1
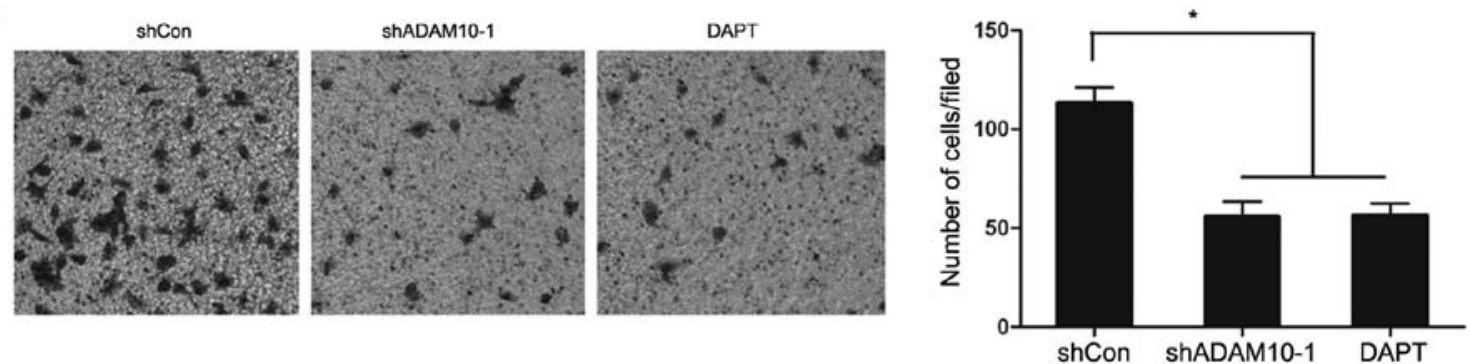

Figure 4. Inhibition of Notch1 signaling suppresses the migration and invasion of NSCLC cells. (A) Effect of DAPT on A549 cell migration. Monolayer of shCon, shADAM10-1 and A549 cells treated with DAPT were scratched with a pipette tip and were monitored at the beginning of the experiment and at $24 \mathrm{~h}$ for wound channel closure. The cleared area was measured and plotted as the percentage of the original time-point $(0 \mathrm{~h})$. (B) Effect of DAPT on A549 cell invasion. shCon, shADAM10-1 cells and A549 cells treated with DAPT that had invaded through a Matrigel-coated $8 \mu \mathrm{m}$ transwell chamber. After $24 \mathrm{~h}$, the invaded cells on the bottom surface were fixed and counted after staining with crystal violet. Ten random fields were counted. Original magnification, x100. Error bars indicate SD $(\mathrm{n}=3) .{ }^{*} \mathrm{P}<0.05,{ }^{* * *} \mathrm{P}<0.01$.
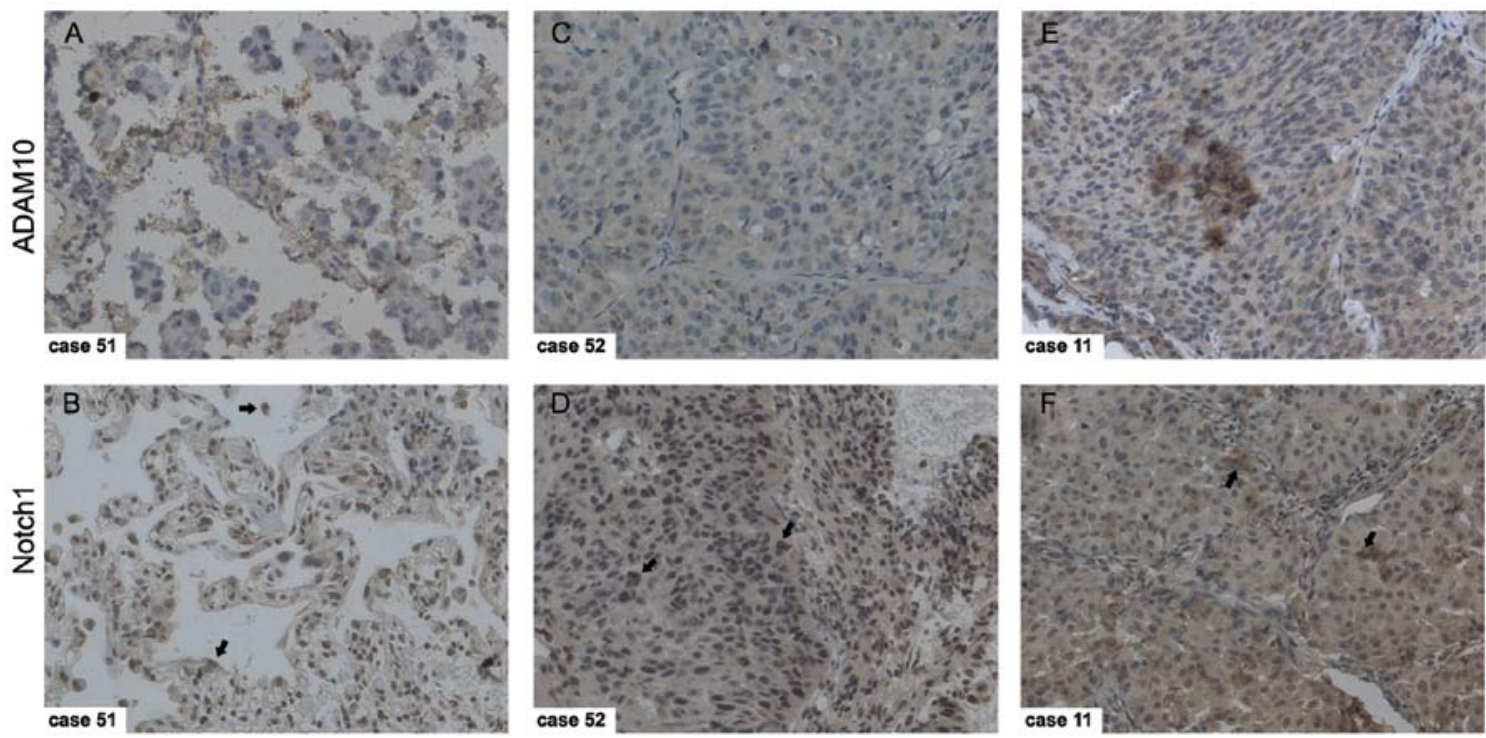

Figure 5. ADAM10 positively correlates with Notch1 expression in NSCLC tissues. (A-D) Two representative NSCLC tissues with ADAM10 weak staining showed low to moderate Notch1 expression; (E-F) NSCLC tissues with strong staining of ADAM10 had a high Notch1 expression. Original magnification, x200.

in both the cytoplasm and nucleus (Fig. 5). It was concluded that ADAM10 expression showed a highly significant positive correlation with Notch1 in the 56 cancer samples (Table II). Taken together, these results suggest that ADAM10 is an endogenous Notch1 activator in NSCLC.

\section{Discussion}

The protease, ADAM10, has been shown to be overexpressed in various malignancies and to influence the progression and metastasis of cancer cells. Gavert et al reported that the expres- 
Table II. Correlation between ADAM10 and Notch1 expression in tumor tissues from 56 patients with NSCLC.

\begin{tabular}{lcccr}
\hline & & \multicolumn{2}{c}{ ADAM10 } & \\
\cline { 3 - 4 } & $\mathrm{n}$ & Low histoscore (0-4) & High histoscore (6-9) & Spearman's test \\
\hline Total & 56 & 25 & 31 & $\mathrm{P}<0.001$ \\
& & & & $\mathrm{r}=0.547$ \\
Notch1 & & 14 & 6 & \\
$\quad$ Low histoscore (0-4) & 20 & 11 & 25 & \\
High histoscore (6-9) & 36 & & & \\
\hline
\end{tabular}

ADAM10, a disintegrin and metalloproteinase, NSCLC, non-small cell lung cancer. Statistical analysis was performed using the Spearman correlation test.

sion of ADAM10 was detected at the invasive front of human colorectal tumor tissues (27). Recently, ADAM10 was detected to be overexpressed in pancreatic cancer and to promote the invasiveness and migration of cancer cells via the inhibition of the cleavage of CXCL16 (9). In adenoid cystic carcinoma, blocking the expression of ADAM10 with ADAM10-specific siRNA inhibited cancer cell proliferation and metastasis (28). For NSCLC, the active form of ADAM10 protein was higher in tumor tissues compared with the corresponding normal tissues from 14 patients. It has been hypothesized that ADAM10 plays a role in angiogenesis and/or metastasis in lung cancer (14). However, reports relating to this hypothesis are very limited. Therefore, in this study, we aimed to investigate the impact of ADAM10 expression on the invasive and metastatic potential of NSCLC cells. We characterized the protein expression of ADAM10 in NSCLC tissues from 56 patients. Immunohistochemical analysis indicated that ADAM10 was overexpressed in malignant tissues compared to matched normal tissues from NSCLC patients. Furthermore, ADAM10 expression was markedly increased in metastatic lymph nodes/ or distant metastatic tissues compared with corresponding primary tumors. Based on the results from clinical correlation analysis and previous reports, it is reasonable to speculate that ADAM10 plays a role in promoting the migration and invasion of NSCLC cells. In this context, we investigated the effects of silencing ADAM10 on in vitro cell migration and invasion. Our results indicated that the knockdown of ADAM10 expression by specific shRNA significantly inhibited the migration and invasion of A549 cells, which have an abnormal Notch expression, and showed very strong ADAM10 protein expression. The data confirm the importance of ADAM10 expression in the process of NSCLC metastasis. The above results support the hypothesis proposed by Zou et al (14).

It is imperative to determine the mechanism by which the knockdown of ADAM10 expression represses the migration and invasion of NSCLC cells. Multiple mechanisms proposed have been associated with the shedding activity of ADAM10 and that it may promote NSCLC progression via the cleavage of its substrates, such as the Notch1 receptor, ERBB2 and E-cadherin to activate the different signaling pathways. We analyzed the protein expression of ERK $1 / 2$ and p-ERK in both ADAM10 knockdown and mock-transfected A549 cells and no significant change was observed. The results are consistent with those from a previous report indicating that INCB3619, the inhibitor of both ADAM10 and 17, induces apoptosis in A549 cells and has antitumor activity in a A549 xenograft model; however, ADAM10 did not play a key role in affecting AKT and ERK activity (14). ADAM17 plays more important roles in activating ErbB signaling. A similar result was obtained for the detection of $\beta$-catenin protein expression in both ADAM10 knockdown and mock-transfected A549 cells. Although we could not rule out the function of $\beta$-catenin in NSCLC cell metastasis, we focused on the Notch1 signaling pathway which is one of the most common signaling pathways contributing to lung cancer $(24,25)$.

The biological role of Notch signaling in cancer was not recognized until 1991 when Notch was suspected to be casually related to the development of T-cell acute lymphocytic leukemia (29). To date, the aberrantly activated Notch1 signaling pathway, an important member of the Notch receptor family, has been observed to mediate tumor metastasis. It has been reported that the downregulation of Notch1 and Jagged1 suppresses the invasion of cervical carcinoma and choriocarcinoma cells (30). Nam et al reported that the $\gamma$-secretase DAPT and RNA interference-mediated knockdown of Notch1 inhibited the migration and invasion of the MDA-MB-435 breast cancer cell line (31). The downregulation of Notch1 inhibited invasion by the inactivation of NF- $\kappa \mathrm{B}, \mathrm{VEGF}$ and MMP-9 in pancreatic cancer cells (32). It was also hypothesized that $\delta$-tocotrienol inhibited cell migration and invasion via inactivation of the Notch1 signaling pathway in NSCLC cells (22). Of note, the increased expression of Notch1 protein was examined in patient tissues and NSCLC stem cells which suggests a role of the Notch1 signaling pathway in NSCLC progression $(25,33)$.

ADAM10 and ADAM17 was identified in previous studies as the major sheddase of the Notch receptor $(34,35)$. Specifically, it was shown that ADAM10 was absolutely required for Notch1 signaling induced by ligands, while signaling independent of ligands required ADAM17 (36). In A549 cells, there was a very strong Notch ligand Jagged1 protein expression (14). A previous report showed that the Notch ligand Jagged 2 promoted lung adenocarcinoma metastasis, but the corresponding Notch receptor remains unknown (19). Based on the above reports, we speculated that ADAM10 may be involved in the cleavage of the Notch1 receptor in NSCLC. First, we detected Notch1 and 
ADAM10 protein co-expression in both tumor and matched normal tissues from NSCLC patients with IHC and the results showed that there was a significantly positive correlation between Notch1 and ADAM10 protein expression. Moreover, the nuclear expression of Notch1 was also observed in several tumor tissues. Second, the effects of silencing ADAM10 on the shedding of Notch1 were also analyzed in vitro. Of note, we found that the knockdown of ADAM10 significantly inhibited the cleavage of NICD which is the activated form of Notch1 in NSCLC cells, using both western blot analysis and immunofluorescence assay. This effect decreased the amount of NICD in the nucleus, which regulates the downstream gene expression. Our results suggest that silencing ADAM10 expression represses the migration and invasion of NSCLC cells by disrupting Notch1 activation. Our findings support a manifest paradigm that the Notch1 signaling pathway activated by ADAM10 shedding plays an important role in the metastasis of NSCLC.

However, the detailed mechanism by which Notch1 signaling influences cancer cell migration and invasion remains unknown. Recently, Xie et al demonstrated that the activation of Notch1 enhanced epithelial mesenchymal transition (EMT) in gefitinib-acquired resistant lung cancer cells (37). Their results indicated that NICD promoted the EMT phenotype by inhibiting the expression of E-cadherin in gefitinib-resistant PC9/AB2 lung cancer cells. Their results suggested that the activation of the Notch1 signaling pathway is critical in gefitinib-acquired resistance and the EMT phenotype in lung adenocarcinoma cells. Their results support our conclusion that ADAM10 promotes migration and invasion via the activation of the Notch1 signaling pathway and may facilitate EMT. The A549 NSCLC cell line is a type of gefitinib resistance cancer cell showing very low E-cadherin expression. Thus, the inhibition of Notch1 may be a novel strategy for the reversal of the EMT phenotype, thereby potentially increasing the therapeutic drug sensitivity of lung cancer cells. McGowan et al recently reported that Notch1 inhibition reduced the formation of brain metastasis from breast cancer via reducing the proportion of the cancer stem cell (CSC) surface markers, CD44hi/CD24lo (38). CSCs are thought to be responsible for the failure of the current chemotherapy of lung cancer (15). There has been a growing body of evidence underscoring the importance of Notch signaling which may impact the CSC phenotype and tumorigenicity $(39,40)$. For example, in $\mathrm{ALDH}^{+}$lung cancer cells (lung CSCs), the elevated expression of Notch1, Notch2 and Notch3 was also observed and the decreased protein expression of the Notch signaling downstream genes, such as Hes1, Hey1 and Hey2 was also detected while treatment with GSIs (33). Further studies are required to determine the underlined mechanism of the inhibition of NSCLC cell metastasis via the inactivation of the Notch1 signaling pathway. In conclusion, our results suggest that the knockdown of ADAM10 by using RNAi technology is not only an experimental technology, but may also serve as a therapeutic tool for the treatment of NSCLC metastasis by targeting CSCs.

Patients with NSCLC have a poor prognosis due to metastasis and drug resistance. EMT plays a role in EGFR-TKI acquired resistant lung adenocarcinoma and cancer metastasis. Notch signaling is emerging as a potentially invaluable molecular pathway to affect NSCLC stem cells and acting as a target for novel NSCLC treatment options (33). Based on previous reports and our results, the Notch1 signaling pathway may be inhibited using different strategies. GSIs, working as a doubleedged sword, are anticancer agents being tested in clinical trials. Since GSIs may cause abnormalities in the gastrointestinal tract, thymus and spleen in rodents (41), their toxicity to normal tissues has attracted increasing attention. Several studies have highlighted the potential of targeting ADAMs as a new approach for anticancer therapy. In fact, ADAM inhibitors are also in preclinical development and ADAM inhibitors seem to have less toxicity than GSIs. Standard chemotherapy in combination with ADAM10 or Notch1 targeting may eliminate both bulk tumor cells and CSCs, and therefore be considered a curative therapy.

In conclusion, we demonstrate that ADAM10 may serve as a potential target for the therapeutic intervention of NSCLC metastasis. This molecule, combined with the Notch1 protein, was significantly co-overexpressed in most NSCLC tissues we examined. Importantly, we provide preclinical evidence for the specific silencing of ADAM10 expression with ADAM10 shRNA as an therapeutic strategy against NSCLC metastasis by targeting Notch1 signaling. Additional extensive research must be implemented on this topic in order to further intensify the understanding of the correlation between ADAM10 and NSCLC cancer progression.

\section{Acknowledgements}

This study was supported by the Doctor Fund Project of the Chinese Ministry of Education (No. 20090142110014) and the Doctor Innovation Fund of the Huazhong University of Science and Technology.

\section{References}

1. Wu X, Piper-Hunter MG, Crawford M, Nuovo GJ, Marsh CB, Otterson GA and Nana-Sinkam SP: MicroRNAs in the pathogenesis of lung cancer. J Thorac Oncol 4: 1028-1034, 2009.

2. Ramalingam S and Belani C: Systemic chemotherapy for advanced non-small cell lung cancer: recent advances and future directions. Oncologist 15: 5-13, 2008.

3. Curran WJ Jr: Treatment of locally advanced non-small cell lung cancer: what we have and have not learned over the past decade. Semin Oncol 32: S2-S5, 2005.

4. Edwards DR, Handsley MM and Pennington CJ: The ADAM metalloproteinases. Mol Aspects Med 29: 258-289, 2008.

5. Duffy MJ, McKiernan E, O'Donovan N and McGowan PM: Role of ADAMs in cancer formation and progression. Clin Cancer Res 15: 1140-1144, 2009.

6. Saftig P and Reissb K: The 'A Disintegrin and metalloproteases' ADAM10 and ADAM17: novel drug targets with therapeutic potential? Eur J Cell Biol 90: 527-535, 2011.

7. Shintani Y, Higashiyama S and Ohta M, et al: Overexpression of ADAM9 in non-small cell lung cancer. Cancer Res 64: 4190-4196, 2004.

8. Tsai WC, Hsu PW, Lai TC, et al: MicroRNA-122, a tumor suppressor microRNA that regulates intrahepatic metastasis of hepatocellular carcinoma. Hepatology 49: 1571-1582, 2009.

9. Gaida MM, Haag N, Günther F, Tschaharganeh DF, Schirmacher P Friess H, Giese NA, Schmidt J and Wente MN: Expression of A disintegrin and metalloprotease 10 in pancreatic carcinoma. Int J Mol Med 26: 281-288, 2010.

10. Crawford HC, Dempsey PJ, Brown G, Adam L and Moss ML: ADAM10 as a therapeutic target for cancer and inflammation. Curr Pharm Des 15: 2288-2299, 2009.

11. Ko SY, Lin SC, Wong YK, Liu CJ, Chang KW and Liu TY: Increase of disintergin metalloprotease 10 (ADAM10) expression in oral squamous cell carcinoma. Cancer Lett 245: 33-43, 2007. 
12. Fogel M, Gutwein $\mathrm{P}$ and Mechtersheimer S, et al: L1 expression as a predictor of progression and survival in patients with uterine and ovarian carcinomas. Lancet 362: 869-875, 2003.

13. Lee SB, Schramme A and Doberstein K, et al: ADAM10 is upregulated in melanoma metastasis compared with primary melanoma. J Invest Dermatol 130: 763-773, 2010.

14. Zhou BB, Peyton M and He B, et al: Targeting ADAM-mediated ligand cleavage to inhibit HER3 and EGFR pathways in non-small cell lung cancer. Cancer Cell 10: 39-50, 2006.

15. Galluzzo P and Bochetta M: Notch signaling in lung cancer. Expert Rev Anticancer Ther 11: 533-540, 2011.

16. López-Malpartida AV, Ludeña MD, Varela G and García Pichel J: Differential ErbB receptor expression and intracellular signaling activity in lung adenocarcinomas and squamous cell carcinomas. Lung Cancer 65: 25-33, 2009.

17. Pelosi G, Scarpa A, Puppa G, et al: Alteration of the E-cadherin/ $\beta$-catenin cell adhesion system is common in pulmonary neuroendocrine tumors and is an independent predictor of lymph node metastasis in atypical carcinoids. Cancer 103: 1154-1164, 2005.

18. Lai EC: Notch signaling: control of cell communication and cell fate. Development 131: 965-973, 2004.

19. Yang Y, Ahn YH, Gibbons DL, et al: The Notch ligand Jagged2 promotes lung adenocarcinoma metastasis through a miR-200dependent pathway in mice. J Clin Invest 121: 1373-1385, 2011.

20. Konishi J, Kawaguchi KS, Vo H, Haruki N, Gonzalez A, Carbone DP and Dang TP: $\gamma$-secretase inhibitor prevents Notch3 activation and reduces proliferation in human lung cancers. Cancer Res 67: 8051-8057, 2007.

21. Chen Y, Li D, Liu H, et al: Notch-1 signaling facilitates survivin expression in human non-small cell lung cancer cells. Cancer Biol Ther 11: 14-21, 2011.

22. Ji X, Wang Z, Geamanu A, Sarkar FH and Gupta SV: Inhibition of cell growth and induction of apoptosis in non-small cell lung cancer cells by delta-tocotrienol is associated with Notch-1 down-regulation. J Cell Biochem 112: 2773-2783, 2011.

23. Westhoff B, Colaluca IN, D'Ario G, et al: Alterations of the Notch pathway in lung cancer. Proc Natl Acad Sci USA 106: 22293-22298, 2009.

24. Donnem T, Andersen S, Al-Shibli K, Al-Saad S, Busund LT and Bremnes RM: Prognostic impact of Notch ligands and receptors in nonsmall cell lung cancer. Cancer 116: 5676-5685, 2010.

25. Hartmann D, de Strooper B and Serneels L, et al: The disintegrin/ metalloprotease ADAM 10 is essential for Notch signalling but not for $\alpha$-secretase activity in fibroblasts. Hum Mol Genet 11: 2615-2624, 2002.

26. Miele L: Notch signaling. Clin Cancer Res 12: 1074-1079, 2006.

27. Gavert N, Conacci-Sorrell M, Gast D, Schneider A, Altevogt P, Brabletz T and Ben-Ze'ev A: L1, a novel target of $\beta$-catenin signaling, transforms cells and is expressed at the invasive front of colon cancers. J Cell Biol 168: 633-642, 2005.
28. Xu Q, Liu X, Chen W and Zhang Z: Inhibiting adenoid cystic carcinoma cells growth and metastasis by blocking the expression of ADAM 10 using RNA interference. J Transl Med 8: 136-146, 2010.

29. Grabher C, von Boehmer H and Look AT: Notch 1 activation in the molecular pathogenesis of T-cell acute lymphoblastic leukaemia. Nat Rev Cancer 6: 347-359, 2006.

30. Pang RT, Leung CO, Ye TM, et al: MicroRNA-34a suppresses invasion through downregulation of Notch1 and Jagged1 in cervical carcinoma and choriocarcinoma cells. Carcinogenesis 31: 1037-1044, 2010.

31. Nam DH, Jeon HM, Kim S, et al: Activation of Notch signaling in a xenograft model of brain metastasis. Clin Cancer Res 14: 4059-4066, 2008.

32. Wang Z, Banerjee S, Li Y, et al: Down-regulation of Notch-1 inhibits invasion by inactivation of nuclear factor- $\kappa \mathrm{B}$, vascular endothelial growth factor, and matrix metalloproteinase-9 in pancreatic cancer. Cancer Res 66: 2778-2784, 2006.

33. Sullivan JP, Spinola M, Dodge M, et al: Aldehyde dehydrogenase activity selects for lung adenocarcinoma stem cells dependent on notch signaling. Cancer Res 70: 9937-9948, 2010.

34. van Tetering G, van Diest P, Verlaan I, van der Wall E, Kopan R and Vooijs M: Metalloprotease ADAM10 is required for Notch1 site 2 cleavage. J Biol Chem 284: 31018-31027, 2009.

35. Brou C, Logeat F, Gupta N, et al: A novel proteolytic cleavage involved in Notch signaling: the role of the disintegrin-metalloprotease TACE. Mol Cell 5: 207-216, 2000.

36. Bozkulak EC and Weinmaster G: Selective use of ADAM10 and ADAM17 in activation of Notch1 signaling. Mol Cell Biol 29: 5679-5695, 2009.

37. Xie M, Zhang L, He CS, et al: Activation of Notch-1 enhances epithelial-mesenchymal transition in gefitinib-acquired resistant lung cancer cells. J Cell Biochem 113: 1501-1513, 2012.

38. McGowan PM, Simedrea C, Ribot EJ, et al: Notch1 inhibition alters the $\mathrm{CD} 44 \mathrm{hi} / \mathrm{CD} 24$ lo population and reduces the formation of brain metastases from breast cancer. Mol Cancer Res 9: 834-844, 2011.

39. Bolós V, Blanco M, Medina V, Aparicio G, Díaz-Prado S and Grande E: Notch signaling in cancer stem cells. Clin Transl Oncol 11: 11-19, 2009.

40. Pannuti A, Foreman K, Rizzo P, Osipo C, Golde T, Osborne B and Miele L: Targeting Notch to target cancer stem cells. Clin Cancer Res 16: 3141-3152, 2010.

41. Barten DM, Meredith JE Jr, Zaczek R, Houston JG and Albright CF: Gamma-secretase inhibitors for Alzheimer's disease: balancing efficacy and toxicity. Drugs R D 7: 87-97, 2006. 